\title{
Lovász-Schrijver PSD-operator on Claw-Free Graphs *
}

\author{
Silvia Bianchi ${ }^{1}$, Mariana Escalante $^{1,2}$, Graciela Nasini $^{1,2}$, and Annegret \\ Wagler $^{3}$ \\ \{sbianchi,mariana, nasini\}@fceia.unr.edu.ar, wagler@isima.fr \\ 1 FCEIA, Universidad Nacional de Rosario, Rosario, Argentina \\ 2 CONICET \\ 3 LIMOS (UMR 6158 CNRS), University Blaise Pascal, Clermont-Ferrand, France
}

\begin{abstract}
The subject of this work is the study of $\mathrm{LS}_{+}$-perfect graphs defined as those graphs $G$ for which the stable set polytope $\operatorname{STAB}(G)$ is achieved in one iteration of Lovász-Schrijver PSD-operator $\mathrm{LS}_{+}$, applied to its edge relaxation $\operatorname{ESTAB}(G)$. In particular, we look for a polyhedral relaxation of $\operatorname{STAB}(G)$ that coincides with $\operatorname{LS}_{+}(\operatorname{ESTAB}(G))$ and $\operatorname{STAB}(G)$ if and only if $G$ is $\mathrm{LS}_{+}$-perfect. An according conjecture has been recently formulated ( $\mathrm{LS}_{+}$-Perfect Graph Conjecture); here we verify it for the well-studied class of claw-free graphs.
\end{abstract}

Keywords: stable set polytope, $\mathrm{LS}_{+}$-perfect graphs, claw-free graphs

\section{Introduction}

The context of this work is the study of the stable set polytope, some of its linear and semi-definite relaxations, and graph classes for which certain relaxations are tight. Our focus lies on those graphs where a single application of the LovászSchrijver positive semi-definite operator introduced in [24] to the edge relaxation yields the stable set polytope.

The stable set polytope $\operatorname{STAB}(G)$ of a graph $G=(V, E)$ is defined as the convex hull of the incidence vectors of all stable sets of $G$ (in a stable set all nodes are mutually nonadjacent). Two canonical relaxations of $\operatorname{STAB}(G)$ are the edge constraint stable set polytope

$$
\operatorname{ESTAB}(G)=\left\{\mathbf{x} \in[0,1]^{V}: x_{i}+x_{j} \leq 1, i j \in E\right\}
$$

and the clique constraint stable set polytope

$$
\operatorname{QSTAB}(G)=\left\{\mathbf{x} \in[0,1]^{V}: \sum_{i \in Q} x_{i} \leq 1, Q \subseteq V \text { maximal clique of } G\right\}
$$

(in a clique all nodes are mutually adjacent, hence a clique and a stable set share at most one node). We have $\operatorname{STAB}(G) \subseteq \operatorname{QSTAB}(G) \subseteq \operatorname{ESTAB}(G)$ for any

\footnotetext{
* This work was supported by a MATH-AmSud cooperation (PACK-COVER), PIDCONICET 0277, and PICT-ANPCyT 0586.
} 
graph, where $\operatorname{STAB}(G)$ equals $\operatorname{ESTAB}(G)$ for bipartite graphs, and $\operatorname{QSTAB}(G)$ for perfect graphs only [5].

According to a famous characterization achieved by Chudnovsky et al. $\underline{3}$, perfect graphs are precisely the graphs without chordless cycles $C_{2 k+1}$ with $k \geq$ 2 , termed odd holes, or their complements, the odd antiholes $\bar{C}_{2 k+1}$ as node induced subgraphs (where the complement $\bar{G}$ has the same nodes as $G$, but two nodes are adjacent in $\bar{G}$ if and only if they are non-adjacent in $G$ ). Then, odd holes and odd antiholes are the only minimally imperfect graphs.

Perfect graphs turned out to be an interesting and important class with a rich structure and a nice algorithmic behavior [19]. However, solving the stable set problem for a perfect graph $G$ by maximizing a linear objective function over $\operatorname{QSTAB}(G)$ does not work directly 18 , but only via a detour involving a geometric representation of graphs [23] and the resulting semi-definite relaxation $\mathrm{TH}(G)$ introduced in [19].

For some $N \in \mathbf{Z}_{+}$, an orthonormal representation of a graph $G=(V, E)$ is a sequence $\left(\mathbf{u}_{\mathbf{i}}: i \in V\right)$ of $|V|$ unit-length vectors $\mathbf{u}_{\mathbf{i}} \in \mathbf{R}^{N}$, such that $\mathbf{u}_{\mathbf{i}}^{T} \mathbf{u}_{\mathbf{j}}=0$ for all $i j \notin E$. For any orthonormal representation of $G$ and any additional unit-length vector $\mathbf{c} \in \mathbf{R}^{N}$, the orthonormal representation constraint is $\sum_{i \in V}\left(\mathbf{c}^{T} \mathbf{u}_{\mathbf{i}}\right)^{2} x_{i} \leq 1 . \mathrm{TH}(G)$ denotes the convex set of all vectors $\mathbf{x} \in \mathbf{R}_{+}^{|V|}$ satisfying all orthonormal representation constraints for $G$. For any graph $G$,

$$
\operatorname{STAB}(G) \subseteq \operatorname{TH}(G) \subseteq \operatorname{QSTAB}(G)
$$

holds and approximating a linear objective function over $\operatorname{TH}(G)$ can be done with arbitrary precision in polynomial time [19]. Moreover, if $\operatorname{TH}(G)$ is a rational polytope, an optimal solution can be obtained in polynomial time. This fact gives a great relevance to the beautiful characterization of perfect graphs obtained by the same authors:

$$
G \text { is perfect } \Leftrightarrow \operatorname{TH}(G)=\operatorname{STAB}(G) \Leftrightarrow \operatorname{TH}(G)=\operatorname{QSTAB}(G) .
$$

For all imperfect graphs, $\operatorname{STAB}(G)$ does not coincide with any of the above relaxations. It is, thus, natural to study further relaxations and to combinatorially characterize those graphs where $\operatorname{STAB}(G)$ equals one of them.

Linear relaxations and related graphs. A natural generalization of the clique constraints are rank constraints associated with arbitrary induced subgraphs $G^{\prime} \subseteq G$. By the choice of the right hand side $\alpha\left(G^{\prime}\right)$, denoting the size of a largest stable set in $G^{\prime}$, rank constraints

$$
\mathbf{x}\left(G^{\prime}\right)=\sum_{i \in G^{\prime}} x_{i} \leq \alpha\left(G^{\prime}\right)
$$

are valid for $\operatorname{STAB}(G)$.

A graph $G$ is called rank-perfect by 34 if and only if $\operatorname{STAB}(G)$ is described by rank constraints only. 
By definition, rank-perfect graphs include all perfect graphs. By restricting the facet set to rank constraints associated with certain subgraphs, several wellknown graph classes are defined, e.g., near-perfect graphs 31] where only rank constraints associated with cliques and the whole graph are allowed, or t-perfect [5] and $h$-perfect graphs [19] where rank constraints associated with edges, triangles and odd holes resp. cliques of arbitrary size and odd holes suffice.

As common generalization of perfect, t-perfect, and h-perfect graphs, the class of a-perfect graphs was introduced in [35] as graphs $G$ where $\operatorname{STAB}(G)$ is given by rank constraints associated with antiwebs. An antiweb $A_{n}^{k}$ is a graph with $n$ nodes $0, \ldots, n-1$ and edges $i j$ if and only if $k \leq|i-j| \leq n-k$ and $i \neq j$. Antiwebs include all complete graphs $K_{n}=A_{n}^{1}$, odd holes $C_{2 k+1}=A_{2 k+1}^{k}$, and their complements $\bar{C}_{2 k+1}=A_{2 k+1}^{2}$. Antiwebs are $a$-perfect by [35], further examples of $a$-perfect graphs were found in 36 .

A more general type of inequalities is obtained from complete joins of antiwebs, called joined antiweb constraints

$$
\sum_{i \leq k} \frac{1}{\alpha\left(A_{i}\right)} x\left(A_{i}\right)+x(Q) \leq 1,
$$

associated with the join of some antiwebs $A_{1}, \ldots, A_{k}$ and a clique $Q$ (note that the inequality is scaled to have right hand side 1). This includes, e.g., all odd (anti)wheels (the join of a single node with an odd (anti)hole). We denote the linear relaxation of $\operatorname{STAB}(G)$ obtained by all joined antiweb constraints by $\operatorname{ASTAB}^{*}(G)$. By construction, we see that

$$
\operatorname{STAB}(G) \subseteq \operatorname{ASTAB}^{*}(G) \subseteq \operatorname{QSTAB}(G) \subseteq \operatorname{ESTAB}(G) .
$$

In [6], a graph $G$ is called joined a-perfect if and only if $\operatorname{STAB}(G)$ coincides with $\operatorname{ASTAB}^{*}(G)$. Besides all a-perfect graphs, further examples of joined a-perfect graphs are near-bipartite graphs (where the non-neighbors of every node induce a bipartite graph) due to 32 .

A semi-definite relaxation and $\mathrm{LS}_{+}$-perfect graphs. In the early nineties, Lovász and Schrijver introduced the PSD-operator $\mathrm{LS}_{+}$(called $N_{+}$in [24]) which, applied to $\operatorname{ESTAB}(G)$, generates a positive semi-definite relaxation of $\operatorname{STAB}(G)$ stronger than $\mathrm{TH}(G)$ (see Section 2 for details). In order to simplify the notation we write $\mathrm{LS}_{+}(G)=\mathrm{LS}_{+}(\operatorname{ESTAB}(G))$.

As in the case of perfect graphs, the stable set problem can be solved in polynomial time for the class of graphs for which $\operatorname{LS}_{+}(G)=\operatorname{STAB}(G)$ by 24 . These graphs are called $\mathrm{LS}_{+}-$perfect, and all other graphs $\mathrm{LS}_{+}$-imperfect (note that they are also called $N_{+}$-(im)perfect, see e.g. [1]).

In addition, every subgraph of an $\mathrm{LS}_{+}$-perfect graph is also $\mathrm{LS}_{+}$-perfect. This motivates the definition of minimally $\mathrm{LS}_{+}$-imperfect graphs as the $\mathrm{LS}_{+}$-imperfect graphs whose proper induced subgraphs are all $\mathrm{LS}_{+}$-perfect. The two smallest of such graphs (regarding its number of nodes) were found by [10 and 22] and are depicted in Figure 1. 

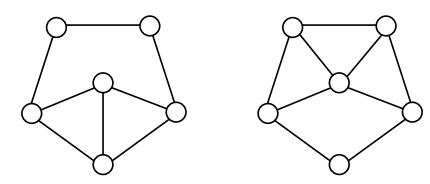

Fig. 1. The graphs $G_{L T}$ (on the left) and $G_{E M N}$ (on the right).

In [1, the authors look for a characterization of $\mathrm{LS}_{+}$-perfect graphs similar to the characterization (11) for perfect graphs: they intend to find an appropriate polyhedral relaxation $P(G)$ of $\operatorname{STAB}(G)$ such that $G$ is $\mathrm{LS}_{+}$-perfect if and only if $\operatorname{STAB}(G)=P(G)$. A conjecture has been recently proposed in [2, which can be equivalently reformulated as follows [12:

Conjecture 1 (LS ${ }_{+}$-Perfect Graph Conjecture). A graph $G$ is $\mathrm{LS}_{+}$-perfect if and only if $\operatorname{STAB}(G)=\operatorname{ASTAB}^{*}(G)$.

In 24] it is shown that, for every graph $G$

$$
\operatorname{LS}_{+}(G) \subseteq \operatorname{ASTAB}^{*}(G) .
$$

Thus, the conjecture states that $\mathrm{LS}_{+}$-perfect graphs coincide with joined aperfect graphs and $\operatorname{ASTAB}^{*}(G)$ is the polyhedral relaxation of $\operatorname{STAB}(G)$ playing the role of $P(G)$ in (11).

Conjecture 1 has been already verified for near-perfect graphs by [1, for $f s$ perfect graphs (where the only facet-defining subgraphs are cliques and the graph itself) by [2], for webs (the complements $W_{n}^{k}=\bar{A}_{n}^{k}$ of antiwebs) by [11] and for line graphs (obtained by turning adjacent edges of a root graph into adjacent nodes of the line graph) by [12, see Section 2 for details.

The $\mathrm{LS}_{+}$-Perfect Graph Conjecture for Claw-free graphs. The aim of this contribution is to verify Conjecture 1 for a well-studied graph class containing all webs, all line graphs and the complements of near-bipartite graphs: the class of claw-free graphs (i.e., the graphs not containing as node induced subgraph the complete join of a single node and a stable set of size three).

Claw-free graphs attracted much attention due to their seemingly asymmetric behavior w.r.t. the stable set problem. On the one hand, the first combinatorial algorithms to solve the problem in polynomial time for claw-free graphs [25130] date back to 1980. Therefore, the polynomial equivalence of optimization and separation due to 19 implies that it is possible to optimize over the stable set polytope of a claw-free graph in polynomial time. On the other hand, the problem of characterizing the stable set polytope of claw-free graphs in terms of an explicit description by means of a facet-defining system, originally posed in [19], was open for several decades. This motivated the study of claw-free graphs and its different subclasses, that finally answered this long-standing problem only recently (see Section 2.2 for details).

The paper is organized as follows: In Section 2, we present the State-of-theArt on $\mathrm{LS}_{+}$-perfect graphs (including families of $\mathrm{LS}_{+}$-imperfect graphs needed 
for the subsequent proofs) and on claw-free graphs, their relevant subclasses and the results concerning the facet-description of their stable set polytopes from the literature. In Section 3, we verify, relying on the previously presented results, Conjecture 1 for the studied subclasses of claw-free graphs. As a conclusion, we obtain as our main result:

Theorem 1. The $\mathrm{LS}_{+}$-Perfect Graph Conjecture is true for all claw-free graphs.

We close with some further remarks and an outlook to future lines of research.

\section{State-of-the-Art}

\subsection{About LS $_{+}$-perfect graphs}

In order to introduce the $\mathrm{LS}_{+}$-operator we denote by $\mathbf{e}_{0}, \mathbf{e}_{1}, \ldots, \mathbf{e}_{n}$ the vectors of the canonical basis of $\mathbf{R}^{n+1}$ (where the first coordinate is indexed zero), $\mathbf{1}$ the vector with all components equal to 1 and $S_{+}^{n}$ the convex cone of symmetric and positive semi-definite $(n \times n)$-matrices with real entries. Let $K \subset[0,1]^{n}$ be a convex set and

$$
\operatorname{cone}(K)=\left\{\left(\begin{array}{c}
x_{0} \\
\mathbf{x}
\end{array}\right) \in \mathbf{R}^{n+1}: \mathbf{x}=x_{0} \mathbf{y} ; \mathbf{y} \in K\right\} .
$$

Then, the convex set $M_{+}(K)$ is defined as:

$$
\begin{aligned}
M_{+}(K)=\left\{Y \in S_{+}^{n+1}:\right. & Y \mathbf{e}_{0}=\operatorname{diag}(Y), \\
& Y \mathbf{e}_{i} \in \operatorname{cone}(K), \\
& \left.Y\left(\mathbf{e}_{0}-\mathbf{e}_{i}\right) \in \operatorname{cone}(K), i=1, \ldots, n\right\},
\end{aligned}
$$

where $\operatorname{diag}(Y)$ denotes the vector whose $i$-th entry is $Y_{i i}$, for every $i=0, \ldots, n$. Projecting this lifting back to the space $\mathbf{R}^{n}$ results in

$$
\operatorname{LS}_{+}(K)=\left\{\mathrm{x} \in[0,1]^{n}:\left(\begin{array}{l}
1 \\
\mathbf{x}
\end{array}\right)=Y \mathbf{e}_{0}, \text { for some } Y \in M_{+}(K)\right\} .
$$

In 24, Lovász and Schrijver proved that $\operatorname{LS}_{+}(K)$ is a relaxation of the convex hull of integer solutions in $K$ and that

$$
\operatorname{LS}_{+}^{n}(K)=\operatorname{conv}\left(K \cap\{0,1\}^{n}\right),
$$

where $\operatorname{LS}_{+}^{0}(K)=K$ and $\operatorname{LS}_{+}^{k}(K)=\operatorname{LS}_{+}\left(\operatorname{LS}_{+}^{k-1}(K)\right)$ for every $k \geq 1$.

In this work we focus on the behavior of a single application of the $\mathrm{LS}_{+-}$ operator to the edge relaxation $\operatorname{ESTAB}(G)$ of the stable set polytope of a graph.

Recall that we write $\operatorname{LS}_{+}(G)=\mathrm{LS}_{+}(\operatorname{ESTAB}(G))$ to simplify the notation and that graphs for which $\mathrm{LS}_{+}(G)=\operatorname{STAB}(G)$ holds are $\mathrm{LS}_{+}$-perfect.

Exhibiting one $\mathrm{LS}_{+}$-imperfect subgraph $G^{\prime}$ in a graph $G$ certifies the $\mathrm{LS}_{+-}$ imperfection of $G$. Hereby, characterizing $\mathrm{LS}_{+}$-imperfect graphs within a certain graph class turns out to be a way to attack the conjecture for this class. 
Recall that $G_{L T}$ and $G_{E M N}$ are the smallest $\mathrm{LS}_{+}$-imperfect graphs. In [1] the authors showed that they are the two smallest members of an infinite family of $\mathrm{LS}_{+}$-imperfect graphs having stability number two that will play a central role in some subsequent proofs:

Theorem 2 ([1] $)$. Let $G$ be a graph with $\alpha(G)=2$ such that $G-v$ is an odd antihole for some node $v . G$ is $\mathrm{LS}_{+}$-perfect if and only if $v$ is completely joined to $G-v$.

Further $\mathrm{LS}_{+}$-imperfect graphs can be obtained by applying operations preserving $\mathrm{LS}_{+}$-imperfection.

In [22, the stretching of a node $v$ is introduced as follows: Partition its neighborhood $N(v)$ into two nonempty, disjoint sets $A_{1}$ and $A_{2}$ (so $A_{1} \cup A_{2}=$ $N(v)$, and $\left.A_{1} \cap A_{2}=\emptyset\right)$. A stretching of $v$ is obtained by replacing $v$ by two adjacent nodes $v_{1}$ and $v_{2}$, joining $v_{i}$ with every node in $A_{i}$ for $i \in\{1,2\}$, and subdividing the edge $v_{1} v_{2}$ by one node $w$. In [22] it is shown:

Theorem 3 ([22]). The stretching of a node preserves $\mathrm{LS}_{+}$-imperfection.

Hence, all stretchings of $G_{L T}$ and $G_{E M N}$ are $\mathrm{LS}_{+}$-imperfect, see Figure 2 for some examples.
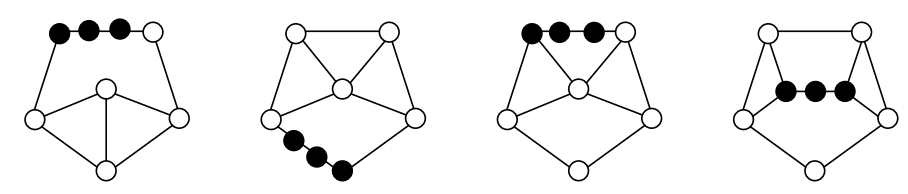

Fig. 2. Some node stretchings $\left(v_{1}, w, v_{2}\right.$ in black) of $G_{L T}$ and $G_{E M N}$.

Using stretchings of $G_{L T}$ and $G_{E M N}$ and exhibiting one more minimally $\mathrm{LS}_{+}$-imperfect graph, namely the web $W_{10}^{2}, \mathrm{LS}_{+}$-perfect webs are characterized in [11] as follows:

Theorem 4 ([11]). A web is $\mathrm{LS}_{+}-$perfect if and only if it is perfect or minimally imperfect.

The proof shows that all imperfect not minimally imperfect webs with stability number 2 contain $G_{E M N}$ and all webs $W_{n}^{2}$ different from $W_{7}^{2}, W_{10}^{2}$, some stretching of $G_{L T}$. Furthermore, all other webs contain some LS $S_{+}$-imperfect $W_{n^{\prime}}^{2}$ and are, thus, also $\mathrm{LS}_{+}$-imperfect.

Another way to attack the conjecture is from the polyhedral point of view.

A graph $G$ is said to be facet-defining if $\operatorname{STAB}(G)$ has a full-support facet. Observe that verifying Conjecture 1 is equivalent to prove that the only facet defining $\mathrm{LS}_{+}$-perfect graphs are the complete joins of antiwebs. That is why we 
need to rely on structural results and complete facet-descriptions of stable set polytope of the graphs.

Using this approach, in [12, the authors characterized $\mathrm{LS}_{+}$-perfect line graphs by showing:

Theorem 5 ([12]). A facet-defining line graph $G$ is $\mathrm{LS}_{+}$-perfect if and only if $G$ is a clique or an odd hole.

The proof relies on a result due to Edmonds \& Pulleyblank [8] who showed that a line graph $L(H)$ is facet-defining if and only if $H$ is a 2-connected hypomatchable graph (that is, for all nodes $v$ of $H, H-v$ admits a perfect matching). Such graphs $H$ have an ear decomposition $H_{0}, H_{1}, \ldots, H_{k}=H$ where $H_{0}$ is an odd hole and $H_{i}$ is obtained from $H_{i-1}$ by adding an odd path (ear) between distinct nodes of $H_{i-1}$. In [12, it is shown that the line graph $L\left(H_{1}\right)$ is a node stretching of $G_{L T}$ or $G_{E M N}$ and, thus, LS $\mathrm{L}_{+}$-imperfect by [22].

Moreover, it is proved that the only minimally $\mathrm{LS}_{+}$-imperfect line graphs are stretchings of $G_{L T}$ and $G_{E M N}$.

\subsection{About claw-free graphs}

In several respects, claw-free graphs are generalizations of line graphs. An intermediate class between line graphs and claw-free graphs form quasi-line graphs, where the neighborhood of any node can be partitioned into two cliques (i.e., quasi-line graphs are the complements of near-bipartite graphs).

Quasi-line graphs can be divided into two subclasses: fuzzy circular interval graphs and semi-line graphs.

Let $\mathcal{C}$ be a circle, $\mathcal{I}$ a collection of intervals in $\mathcal{C}$ without proper containments and common endpoints, and $V$ a multiset of points in $\mathcal{C}$. A fuzzy circular interval graph $G(V, \mathcal{I})$ has node set $V$ and two nodes are adjacent if both belong to one interval $I \in \mathcal{I}$, where edges between different endpoints of the same interval may be omitted.

Semi-line graphs are either line graphs or quasi-line graphs without a representation as a fuzzy circular interval graph.

It turned out that so-called clique family inequalities suffice to describe the stable set polytope of quasi-line graphs. Given a graph $G$, a family $\mathcal{F}$ of cliques and an integer $p<n=|\mathcal{F}|$, the clique family inequality $(\mathcal{F}, p)$ is the following valid inequality for $\operatorname{STAB}(G)$

$$
(p-r) \sum_{i \in W} x_{i}+(p-r-1) \sum_{i \in W_{o}} x_{i} \leq(p-r)\left\lfloor\frac{n}{p}\right\rfloor
$$

where $r=n \bmod p$ and $W$ (resp. $\left.W_{o}\right)$ is the set of nodes contained in at least $p$ (resp. exactly $p-1$ ) cliques of $\mathcal{F}$.

This generalizes the results of Edmods [7] and Edmonds \& Pulleyblank 8] that $\operatorname{STAB}(L(H))$ is described by clique constraints and rank constraints

$$
x\left(L\left(H^{\prime}\right)\right) \leq \frac{1}{2}\left(\left|V\left(H^{\prime}\right)\right|-1\right)
$$


associated with the line graphs of 2-connected hypomatchable induced subgraphs $H^{\prime} \subseteq H$. Note that the rank constraints of type (4) are special clique family inequalities.

Chudnovsky and Seymour [4 extended this result to semi-line graphs, for which $\operatorname{STAB}(G)$ is given by clique constraints and rank constraints of type (4). Then, semi-line graphs are rank-perfect with line graphs as only facet-defining subgraphs.

Moreover, in [16] Galluccio and Sassano prove that if a rank constraint is facet-defining for a claw-free graph $G$ then, either, $G$ is a clique or $G$ contains the line graph of a minimal 2-connected hypomatchable graph $H$ or $G$ contains $W_{\alpha k+1}^{k-1}$ with $k \geq 3$ and $\alpha=\alpha(G)$.

Eisenbrand et al. 9] proved that clique family inequalities suffice to describe the stable set polytope of fuzzy circular interval graphs. Stauffer [33 verified a conjecture of [27] that every facet-defining clique family inequality of a fuzzy circular interval graph $G$ is associated with a web in $G$.

All these results together complete the picture for quasi-line graphs.

However, there are claw-free graphs which are not quasi-line. In particular, every graph with stability number 2 is claw-free and the 5 -wheel is the smallest claw-free not quasi-line graph.

Due to Cook (see [32]), all facets for graphs $G$ with $\alpha(G)=2$ are 1,2valued clique-neighborhood constraints. This is not the case for graphs $G$ with $\alpha(G)=3$. In fact, all the known difficult facets of claw-free graphs occur in this class. Some non-rank facets with up to five different non-zero coefficients are presented in [17/21. All of these facets turned out to be so-called co-spanning 1-forest constraints due to [28, where it is also shown that it is possible to build a claw-free graph with stability number three inducing a co-spanning 1-forest facet with $b$ different left hand side coefficients, for every positive integer $b$.

The problem of characterizing $\operatorname{STAB}(G)$ when $G$ is a connected claw-free but not quasi-line graph with $\alpha(G) \geq 4$ was studied by Galluccio et al.: In a series of results [13|14|15], it is shown that if such a graph $G$ does not contain a clique cutset, then 1,2-valued constraints suffice to describe $\operatorname{STAB}(G)$. Here, besides 5 -wheels, different rank and non-rank facet-defining inequalities of the geared graph $G$ shown in Fig. 3 play a central role.

In addition, graphs of this type can be decomposed into strips. A strip $(G, a, b)$ is a (not necessarily connected) graph with two designated simplicial nodes $a$ and $b$ (a node is simplicial if its neighborhood is a clique). A claw-free strip containing a 5 -wheel as induced subgraph is a 5-wheel strip. Given two node-disjoint strips $\left(G_{1}, a_{1}, b_{1}\right)$ and $\left(G_{2}, a_{2}, b_{2}\right)$, their composition is the union of $G_{1} \backslash\left\{a_{1}, b_{1}\right\}$ and $G_{2} \backslash\left\{a_{2}, b_{2}\right\}$ together with all edges between $N_{G_{1}}\left(a_{1}\right)$ and $N_{G_{2}}\left(a_{2}\right)$, and between $N_{G_{1}}\left(b_{1}\right)$ and $N_{G_{2}}\left(b_{2}\right)$ [4.

As shown in 26, this composition operation can be generalized to more than two strips: Every claw-free but not quasi-line graph $G$ with $\alpha(G) \geq 4$ admits a decomposition into strips, where at most one strip is quasi-line and all the remaining ones are 5-wheel strips having stability number at most 3 . There are 
only three "basic" types of 5 -wheel strips (see Fig. 31) which can be extended by adding nodes belonging to the neighborhood of the 5 -wheels (see [26] for details).

Note that a claw-free but not quasi-line graph $G$ with $\alpha(G) \geq 4$ containing a clique cutset may have a facet-defining subgraph $G^{\prime}$ with $\alpha\left(G^{\prime}\right)=3$ (inside a 5 -wheel strip of type 3), see [29] for examples.

Taking all these results together into account gives the complete list of facets needed to describe the stable set polytope of claw-free graphs.

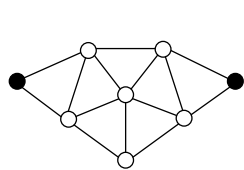

type 1

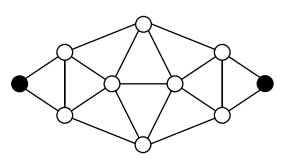

type 2 (gear)

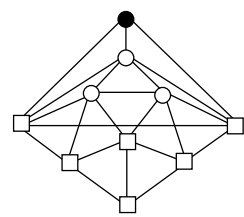

type 3

Fig. 3. The three types of basic 5-wheel strips.

\section{$3 \quad \mathrm{LS}_{+}$-Perfect Graph Conjecture for claw-free graphs}

In this section, we verify the $\mathrm{LS}_{+}$-Perfect Graph Conjecture for all relevant subclasses of claw-free graphs.

\subsection{Graphs with $\alpha(G)=2$}

The graphs with $\alpha(G)=2$ play a crucial role in this context. Relying on the behavior of the stable set polytope under taking complete joins [5] and the result on $\mathrm{LS}_{+}-(\mathrm{im})$ perfect graphs $G$ with $\alpha(G)=2$ (Theorem 2), we can prove:

Theorem 6. All facet-defining $\mathrm{LS}_{+}$-perfect graphs $G$ with $\alpha(G)=2$ are odd antiholes or complete joins of odd antihole(s) and a (possibly empty) clique.

Proof. Let $G$ be a facet-defining $\mathrm{LS}_{+}$-perfect graph with stability number 2 . We first observe that $G$ is imperfect (because it has a full-support facet, but it is different from a clique). Thus, $G$ contains an odd antihole $\bar{C}$ by 3 .

If $G=\bar{C}$, we are done. If $G \neq \bar{C}$, every node $u$ outside $\bar{C}$ is completely joined to $\bar{C}$ due to Theorem 2 (otherwise, $u$ and $\bar{C}$ induce an $\mathrm{LS}_{+}$-imperfect subgraph of $G$, a contradiction to $G \mathrm{LS}_{+}$-perfect).

Therefore, $G$ is the complete join of $\bar{C}$ and $G-\bar{C}$. Note that $G-\bar{C}$ is again $\mathrm{LS}_{+}$-perfect, facet-defining by Chvátal [5, and $\alpha(G-\bar{C}) \leq 2$. If $\alpha(G-\bar{C})=2$, we apply the same argument as for $G$; if $\alpha(G-\bar{C})=1$, it is a clique.

This shows that all facet-defining $\mathrm{LS}_{+}$-perfect graphs $G$ with $\alpha(G)=2$ are joined $a$-perfect, and we conclude: 
Corollary 1. The $\mathrm{LS}_{+}$-Perfect Graph Conjecture is true for graphs with stability number 2.

\subsection{Quasi-line graphs}

Recall that quasi-line graphs divide into the two subclasses of semi-line graphs and fuzzy circular interval graphs.

Chudnovsky and Seymour 4 proved that the stable set polytope of a semiline graph is given by rank constraints associated with cliques and the line graphs of 2-connected hypomatchable graphs. Together with the result from 12] (presented in Theorem 5), we directly conclude that the $\mathrm{LS}_{+}$-Perfect Graph Conjecture holds for semi-line graphs.

Based on the results of Eisenbrand et al. [9] and Stauffer [33, combined with the characterization of $\mathrm{LS}_{+}$-imperfect webs from [1] (Theorem 44), we are able to show:

Theorem 7. All facet-defining $\mathrm{LS}_{+}$-perfect fuzzy circular interval graphs are cliques, odd holes or odd antiholes.

Proof. Let $G$ be a fuzzy circular interval graph such that it is a facet-defining $\mathrm{LS}_{+}$-perfect graph. If $G$ is a clique, the result is immediate. Otherwise, $G$ is the support graph of a clique family inequality with parameters $(\mathcal{F}, p)$

$$
(p-r) \sum_{i \in W} x_{i}+(p-r-1) \sum_{i \in W_{o}} x_{i} \leq(p-r)\left\lfloor\frac{n}{p}\right\rfloor,
$$

associated with a web $W_{n}^{p-1}$ with $V\left(W_{n}^{p-1}\right) \subset W(9[33)$.

More precisely, if for any node $v \in V, \mathcal{I}_{v}=\{I \in \mathcal{I}: v \in I\}$, there exist $I_{l}(v)$ and $I_{r}(v)$ in $\mathcal{I}_{v}$ such that $I_{l}(v) \cup I_{r}(v)=\bigcup_{I \in \mathcal{I}_{v}} I$. The clique family inequality associated with $W_{n}^{p-1}$ is the clique family inequality having parameters $\mathcal{F}=\left\{K\left(I_{l}(v)\right): v \in V\left(W_{n}^{p-1}\right)\right\}$ and $p$ where $K\left(I_{l}(v)\right)=\left\{u \in I_{l}(v)\right.$ : $u$ is adjacent to $v\}$.

By Theorem 4, $W_{n}^{p-1}$ is $\mathrm{LS}_{+}$-perfect if and only if it is an odd hole or an odd antihole.

That is, since $G$ is $\mathrm{LS}_{+}$-perfect then $n=2 k+1$ and $p=2$ or $p=k \geq 3$. In both cases, $r=1$ follows.

Consider first the case in which $p=2$. Then the clique family inequality $(\mathcal{F}, p)$ takes the form

$$
\sum_{i \in W} x_{i} \leq\left\lfloor\frac{n}{p}\right\rfloor .
$$

Suppose there exists $v \in W \backslash V\left(W_{2 k+1}^{1}\right)$. Then, $v$ belongs to $s \geq 2$ consecutive cliques in $\mathcal{F}$ implying that $v$ is connected to exactly $s+1$ consecutive nodes in $W_{2 k+1}^{1}$. Observe that $s \leq 3$ since $G$ is a claw-free graph. Then, if $s=2$ (resp. $s=3) G$ contains an odd subdivision of $G_{L T}\left(\right.$ resp. $\left.G_{E M N}\right)$. Since $G$ is $L S_{+^{-}}$ perfect then $W=V\left(W_{2 k+1}^{1}\right)$ or, equivalently, $G=W_{2 k+1}^{1}$. 
Now suppose that $p=k \geq 3$. Let us call $\{1,2, \ldots, 2 k+1\}$ the nodes in $V\left(W_{2 k+1}^{k-1}\right)$.

Suppose there exists $v \in\left(W_{o} \cup W\right) \backslash V\left(W_{2 k+1}^{k-1}\right)$. Then $v$ belongs to at least $s \geq k-1$ consecutive cliques of the family $\mathcal{F}$. W.l.o.g we may assume that the $k-1$ of the $s$ consecutive cliques are the ones that contain the sets of nodes $\{1, \ldots, k\}$, $\{2, \ldots, k+1\}, \ldots\{k-1, \ldots, 2 k-2\}$. Then $v$ is connected to at least $2 k-2$ consecutive nodes in $W_{2 k+1}^{k-1}$. Moreover, since $G$ is quasi-line, $v$ is connected with at most $2 k$ nodes. It follows that the subgraph of $G$ induced by $V\left(W_{2 k+1}^{k-1}\right) \cup\{v\}$ has stability number two, and from Theorem [6] it is $\mathrm{LS}_{+}$-imperfect. But from our assumption that $G$ is $\mathrm{LS}_{+}$-perfect, we conclude $W_{o} \cup W=V\left(W_{2 k+1}^{k-1}\right)$ or, equivalently, $G=W_{2 k+1}^{k-1}$.

As a consequence, every $\mathrm{LS}_{+}$-perfect fuzzy circular interval graph is a-perfect. This verifies the $\mathrm{LS}_{+}$-Perfect Graph Conjecture for fuzzy circular interval graphs.

Since the class of quasi-line graphs divides into semi-line graphs and fuzzy circular interval graphs, we obtain as direct consequence:

Corollary 2. The $\mathrm{LS}_{+}$-Perfect Graph Conjecture is true for quasi-line graphs.

\subsection{Claw-free graphs that are not quasi-line}

It is left to treat the case of claw-free graphs that are not quasi-line. Here, we distinguish two cases according to their stability number.

To treat the case of claw-free not quasi-line graphs $G$ with $\alpha(G) \geq 4$, we rely on the decomposition of such graphs into strips, where at most one strip is quasi-line and all the remaining ones are 5-wheel strips [26]. By noting that 5-wheel strips of type 3 contain $G_{L T}$ and exhibiting $\mathrm{LS}_{+}$-imperfect line graphs in the other two cases, we are able to show:

Theorem 8. Every facet-defining claw-free not quasi-line graph $G$ with $\alpha(G) \geq$ 4 is $\mathrm{LS}_{+}$-imperfect.

Proof. Let $G$ be a facet-defining claw-free not quasi-line graph with $\alpha(G) \geq 4$. According to [26], $G$ has a decomposition into strips, where at most one strip is quasi-line and all the remaining ones have stability number at most 3 and contain a 5-wheel each. Recall that there are only three types of 5-wheel strips, Fig. 3 shows the "basic" types, which can be extended by adding nodes belonging to the neighborhood of the 5 -wheels 26 .

Since $G$ is not quasi-line, it contains at least one 5 -wheel strip $G^{\prime}$. If $G^{\prime}$ is of type 3 , then $G^{\prime}$ contains $G_{L T}$, induced by the squared nodes indicated in Fig. 3 , and we are done. Hence, let $G^{\prime}$ be of type 1 or 2 .

Note further that $G^{\prime}$ is a proper subgraph of $G$ (by $\alpha\left(G^{\prime}\right) \leq 3$ but $\alpha(G) \geq 4$ ) and connected to $G-G^{\prime}$ (since $G$ is facet-defining and, thus, cannot have a clique cutset by Chvátal [5]).

According to the strip composition, there are nodes in $G-G^{\prime}$ playing the role of the two simplicial nodes of $G^{\prime}$ (the two black nodes in Fig. 3), and they 
are connected by a path $P$ with nodes exclusively in $G-G^{\prime}$ (again, since $G$ cannot contain a clique cutset).

If $G^{\prime}$ is of type 1 , then $G$ has, as induced subgraph, a node stretching of

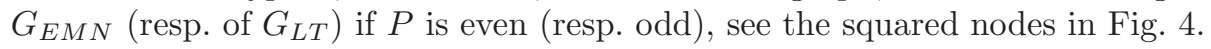
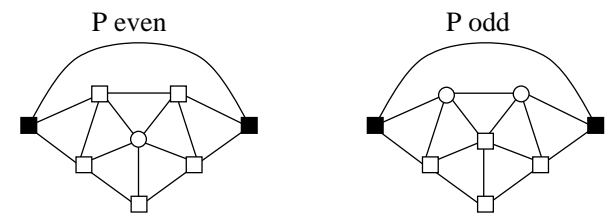

Fig. 4. $N_{+}$-imperfect subgraphs if $G^{\prime}$ is of type 1 .

If $G^{\prime}$ is of type 2, then $G$ has, as induced subgraph, a node stretching of $G_{L T}$ (resp. of $G_{E M N}$ ) if $P$ is even (resp. odd), see the squared nodes in Fig. 5]

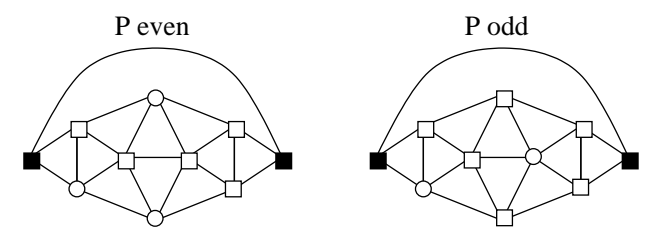

Fig. 5. $\mathrm{LS}_{+}$-imperfect subgraphs if $G^{\prime}$ is of type 2 .

Hence, in all cases, $G$ contains an $\mathrm{LS}_{+}$-imperfect line graph and is itself $\mathrm{LS}_{+}$-imperfect.

For graphs having stability number three, there is no decomposition known yet. Relying only on the behavior of the stable set polytope under clique identification [5] and the result on $\mathrm{LS}_{+}-(\mathrm{im})$ perfect graphs from Theorem 2, we can prove:

Theorem 9. Every facet-defining claw-free not quasi-line graph $G$ with $\alpha(G)=$ 3 is $\mathrm{LS}_{+}$-imperfect.

Proof. Let $G$ be a facet-defining claw-free graph with $\alpha(G)=3$ that is not quasiline. Then, there is a node $v$ in $G$ such that $G^{\prime}=G[N(v)]$ cannot be partitioned into 2 cliques. Hence, in the complement $\bar{G}$ of $G$, the subgraph $\bar{G}^{\prime}$ cannot be partitioned into two stable sets. Thus, $\bar{G}^{\prime}$ is non-bipartite and contains an odd cycle. Let $C$ be the shortest odd cycle in $\bar{G}^{\prime}$. Then $C$ is not a triangle (otherwise, $\bar{C}$ and $v$ induce a claw in $G$ ). Hence, $C$ is an odd hole (because it is an odd cycle of length $\geq 5$, but has no chords according to its choice). 
Therefore, $\bar{C}$ is an odd antihole in $G$; let $u_{1}, \ldots, u_{2 k+1}$ denote it nodes and $u_{i} u_{i+k}$ be its non-edges. Furthermore, let $G_{v}=G[N(v) \cup\{v\}]$ and $W=N(v)-\bar{C}$.

From now on, we will use $\bar{C}$ to denote both, the node set and the odd antihole when it is clear from the context.

Claim $1 G_{v}$ has stability number 2 and

- either contains an $L S_{+}$-imperfect subgraph

- or is the complete join of $v, \bar{C}$ and $W$.

Firstly, note that $N(v)$ does not contain a stable set of size 3 (otherwise, $G$ clearly contains a claw). Hence, $\alpha\left(G_{v}\right)=2$ follows. If $W=\emptyset$, we are done. If there is a node $w \in W$, then for each such node, either $w$ is completely joined to $\bar{C}$ or else the subgraph of $G$ induced by $w$ and $\bar{C}$ is $L S_{+}$-imperfect due to Theorem 2. $\diamond$

We are done if $G_{v}$ is $L S_{+}$-imperfect. Hence, assume in the sequel of this proof that $G_{v}$ is the complete join of $v, \bar{C}$ and $W$. Since $\alpha(G)=3$ holds, $G_{v}$ is a proper subgraph of $G$. We partition the nodes in $G-G_{v}$ into 3 different subsets:

- $X$ containing all nodes from $G-G_{v}$ having a neighbor in $W$,

- $Y$ having all nodes from $G-G_{v}$ having no neighbor in $W$, but a neighbor in $\bar{C}$,

- $Z$ containing all nodes from $G-G_{v}$ having no neighbor in $W \cup \bar{C}$.

Claim 2 Every node $x \in X$

- either induces together with $\bar{C}$ an $L S_{+}$-imperfect subgraph of $G$,

- or is completely joined to $\bar{C}$.

No node $x \in X$ can belong to a stable set $S=\left\{x, u_{i}, u_{i+k}\right\}$ (otherwise, any neighbor $w \in W$ of $x$ induces together with $S$ a claw in $G$ ). Hence, for every $x \in X$, the subgraph $G[\bar{C} \cup\{x\}]$ has stability number 2 and is either $L S_{+^{-}}$ imperfect or an odd antiwheel by Theorem 2 $\diamond$

We are done if some node $x \in X$ yields an $L S_{+}$-imperfect graph $G[\bar{C} \cup\{x\}]$. Hence, assume in the sequel of this proof that $\bar{C}$ and $X$ are completely joined.

Claim $3 X$ is a clique.

Otherwise, $G$ contains a claw containing some node $u_{i} \in \bar{C}$ as central node, $v$ and two non-adjacent nodes $x, x^{\prime} \in X$. $\diamond$

Let $G_{X}$ denote the subgraph of $G$ induced by $v, N(v)$ and $X$.

Claim 4 We have $\alpha\left(G_{X}\right)=2$.

We know already that $\alpha\left(G_{v}\right)=2$ by Claim 1 . If $G_{X}$ contains a stable set $S$ of size 3, then $x \in S$ for some $x \in X$. This implies $S \cap \bar{C}=\emptyset$ (recall that we assume that $X$ and $\bar{C}$ are completely joined). In addition, $v \notin S$ (since $v$ is adjacent to all nodes in $W$ (so we would have $S \cap W=\emptyset$ if $v \in S$ ), but $S$ cannot contain 
2 nodes from $X$ (since $X$ is a clique by Claim 3)). Finally, $S$ cannot contain 2 non-adjacent nodes $w, w^{\prime} \in W$ (otherwise, any node $u_{i} \in \bar{C}$ induces with $S$ a claw in $G$ ). Hence, there is no such stable set $S$ in $G_{X} . \diamond$

By $\alpha(G)=3$ and $\alpha\left(G_{X}\right)=2$, there is a node in $Y \cup Z$. We conclude that $Y \neq \emptyset$ (otherwise, $X$ would constitute a clique cutset of $G$, separating $Z$ from $G^{\prime}$, a contradiction to $G$ facet-defining by Chvátal [5].

Claim $5 W$ induces a clique.

Otherwise, $G$ contains a claw induced by some node $y \in Y$, a neighbor $u_{i} \in \bar{C}$ of $y$, and two non-adjacent nodes $w, w^{\prime} \in W$. $\diamond$

Hence, $G_{v}$ is in fact the complete join of a clique $Q=\{v\} \cup W$ and $\bar{C}$. In addition, $X$ is a clique and completely joined to $\bar{C}, Y$ is non-empty, and there is no edge between $Q$ and $Y$. We further obtain:

Claim 6 Every node $y \in Y$ is completely joined to $X$.

Otherwise, $G$ contains a claw induced by some node $y \in Y$, a neighbor $u_{i} \in \bar{C}$ of $y, v$ and a non-neighbor $x \in X$ of $y$. $\diamond$

Note that, according to Theorem [2 each node $y \in Y$ has three possibilities for its connections to $\bar{C}$ :

- either $y$ induces together with $\bar{C}$ an $L S_{+}$-imperfect subgraph of $G$,

- or $y$ is completely joined to $\bar{C}$,

- or $y$ belongs to a stable set $S_{y}=\left\{y, u_{i}, u_{i+k}\right\}$

(recall that, by Theorem 2, whenever $\{y\} \cup \bar{C}$ has stability number 2, it is either $L S_{+}$-imperfect or an odd antiwheel). If a node $y \in Y$ gives rise to an $L S_{+^{-}}$ imperfect subgraph of $G$, we are done. Hence, assume in the sequel of this proof that $Y$ is partitioned into two subsets $Y_{*}$ and $Y_{S}$ containing all nodes $y$ that are completely joined to $\bar{C}$ resp. belong to a stable set $S_{y}=\left\{y, u_{i}, u_{i+k}\right\}$. We next show:

Claim $7 \quad Y_{S} \neq \emptyset$.

Assume to the contrary that we have $Y=Y_{*}$. Then, $Y$ also induces a clique (otherwise, there is a claw in $G$ induced by $v$, some node $u_{i} \in \bar{C}$ and two nonadjacent nodes $y, y^{\prime} \in Y$ ). This implies that $G\left[G_{v} \cup X \cup Y\right]$ has stability number 2 (by $\alpha\left(G_{X}\right)=2$ due to Claim $4, Y$ completely joined to $X$ due to Claim 6 , and $Y=Y_{*}$ clique). Thus, $Z$ is non-empty (because $\alpha(G)=3$ ). Hence, $G$ contains a clique cutset $X \cup Y$, separating $Z$ from $G_{v}$ (recall that every node in $Z$ has only neighbors in $X$ or $Y$, but not in $G_{v}$ ), a contradiction to $G$ facet-defining by Chvátal [5]. Therefore, we conclude that $Y=Y_{*}$ cannot hold. $\diamond$

Having ensured the existence of a stable set $S_{y}=\left\{y, u_{i}, u_{i+k}\right\}$ in $G$, we next observe: 
Claim $8 \quad X=\emptyset$.

Otherwise, $G$ contains a claw induced by $S_{y}$ and any node $x \in X$ (recall: we assume that $X$ and $\bar{C}$ are completely joined (otherwise, $G$ is $L S_{+}$-imperfect by Claim 2), and have that $X$ and $Y$ are completely joined by Claim 6 ). $\diamond$

This implies particularly that no node outside $G_{v}$ has a neighbor in $W$. We next study the connections between $\bar{C}$ and $Y$ in more detail and obtain the following important fact:

Claim $9 \bar{C}=C_{5}$ and each node $y \in Y_{S}$ has exactly two consecutive neighbors on $\bar{C}$.

Consider some node $y \in Y_{S}$ and the stable set $S_{y}=\left\{y, u_{i}, u_{i+k}\right\}$. By construction of $Y, y$ has a neighbor $u_{j} \in \bar{C}$. This node $u_{j}$ (and any further neighbor of $y$ in $\bar{C}$ ) cannot be a common neighbor of $u_{i}$ and $u_{i+k}$ (otherwise, $u_{j}$ induces together with $S_{y}$ a claw in $G$ ). Hence, $u_{j}$ equals either $u_{i-1}$ (which is not adjacent to $u_{i+k}$ ) or else $u_{i+k+1}$ (which is not adjacent to $u_{i}$ ). W.l.o.g., say that $y$ has $u_{i-1}$ as neighbor in $\bar{C}$. Then $\bar{C}=C_{5}$ follows because for any $k \geq 3$, the graph induced by $y$ and $\bar{C}$ contains a claw with center $u_{i-1}$ and the nodes $u_{i+1}, u_{i+k+2}, y$ (or else $u_{i+1}$ or $u_{i+k+2}$ induce with $S_{y}$ a claw if $y$ is adjacent to $u_{i+1}$ or $u_{i+k+2}$ ).

Moreover, we observe that $y$ is also adjacent to $u_{i-2}$ (otherwise, there is a claw with center $u_{i-1}$ and the nodes $u_{i}, u_{i-2}, y$ ). This shows the assertion that each node $y \in Y_{S}$ has exactly two consecutive neighbors on $\bar{C}=C_{5}$. $\diamond$

We next observe:

Claim $10 Z$ induces a clique.

Otherwise, $G$ contains a stable set of size 4 , consisting of two non-adjacent nodes in $\bar{C}$ and two non-adjacent nodes in $Z$ (recall: by definition of $Z$, there is no edge between $\bar{C}$ and $Z$ ). $\diamond$

Hence, so far we have the following: $G_{v}$ is the complete join of a clique $Q=\{v\} \cup W$ and $\bar{C}=C_{5} . G-G_{v}$ is partitioned into two subsets $Y$ and $Z$ where

- $Y$ is non-empty and partitions into two subsets $Y_{*}$ and $Y_{S}$ consisting of all nodes $y$ that are completely joined to $\bar{C}=C_{5}$ resp. belong to a stable set $S_{y}=\left\{y, u_{i}, u_{i+k}\right\}$ and have exactly two consecutive neighbors on the $C_{5}$;

- $Z$ induces a clique and no node in $Z$ has a neighbor in $G_{v}$.

We continue to explore the composition of $Y$ and its connections to $\bar{C}=C_{5}$ :

Claim 11 If two nodes $y, y^{\prime} \in Y$ share a same neighbor $u_{j} \in \bar{C}$, then $y$ and $y^{\prime}$ are adjacent.

Otherwise, $G$ contains a claw with center $u_{j}$ and the nodes $v, y, y^{\prime} . \diamond$

Claim 12 There are at least two nodes in $Y_{S}$. 
By Claim 7, there is a node $y \in Y_{S}$. Then $Y \neq\{y\}$ follows (otherwise, the only two and consecutive neighbors of $y$ on $\bar{C}=C_{5}$ (by Claim 9) form a clique cutset in $G$, separating $y$ from $Q$, a contradiction to $G$ facet-defining by Chvátal [5]). If all nodes from $Y-\{y\}$ belong to $Y_{*}$, then $Y$ induces a clique by Claim 11 (because all share a common neighbor in $\bar{C}$ ), and $Y-\{y\}$ together with the two and consecutive neighbors of $y$ on $\bar{C}$ form a clique cutset in $G$, separating $y$ from $Q$, again a contradiction to $G$ facet-defining. Hence, $Y_{S}$ contains at least two nodes. $\diamond$

Using similar arguments, we next show:

Claim 13 Not all nodes in $Y_{S}$ have the same two consecutive neighbors on $\bar{C}$.

Otherwise, $G$ has a clique cutset (consisting of $Y_{*}$ and the two common, consecutive neighbors on $\bar{C}$ of all nodes in $Y_{S}$ ), separating $Y_{S}$ from $Q$, again a contradiction to $G$ facet-defining. $\diamond$

Claim 14 If all nodes in $Y_{S}$ share a common neighbor $u_{i}$ on $\bar{C}$, then $G$ contains $G_{E M N}$ as induced subgraph.

By assumption, we have only three types of nodes in $Y$ : nodes $y$ with $N_{\bar{C}}(y)=$ $\left\{u_{i-1}, u_{i}\right\}$, nodes $y^{\prime}$ with $N_{\bar{C}}\left(y^{\prime}\right)=\left\{u_{i}, u_{i+1}\right\}$, and nodes $y_{*} \in Y_{*} . Y$ induces a clique (by Claim 11) and $Z=\emptyset$ follows (otherwise, $Y$ is a clique cutset separating $Z$ from $Q$, again a contradiction to $G$ facet-defining). Claim 12 combined with Claim 13 shows that there is at least one node $y$ adjacent to $u_{i-1}, u_{i}$ and at least one node $y^{\prime}$ adjacent to $u_{i}, u_{i+1}$. Moreover, there is also at least one node $y_{*} \in Y_{*}$ (otherwise, $G$ equals the gear (induced by $v, \bar{C}, y$ and $y^{\prime}$, see Figure 6) with possible replications of $v, y, y^{\prime}$ and is not facet-defining, a contradiction). Hence, $G$ contains $G_{E M N}$ (induced by $y_{*}$ and the nodes $v, u_{i-1}, u_{i+1}, y$, and $y^{\prime}$, see Figure 6). $\diamond$

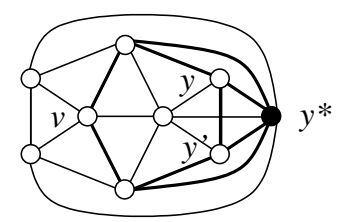

Fig. 6. Subgraph induced by $\bar{C}$ and $v, y, y^{\prime}, y_{*}$ (removing $y_{*}$ yields the gear, the bold edges indicate the $G_{E M N}$ ).

This shows that $G$ is either $L S_{+}$-imperfect (and we are done) or $Y_{S}$ has two nodes with distinct neighbors on $\bar{C}$. Let us assume the latter.

Claim 15 If two nodes $y y^{\prime} \in Y_{S}$ with distinct neighbors on $\bar{C}$ are adjacent, then $G$ contains $G_{L T}$ as induced subgraph. 
W.l.o.g., let $N_{\bar{C}}(y)=\left\{u_{1}, u_{2}\right\}$ and $N_{\bar{C}}\left(y^{\prime}\right)=\left\{u_{3}, u_{4}\right\}$. If $y$ is adjacent to $y^{\prime}$, then $u_{1}, y, y^{\prime}, u_{4}, u_{5}$ induce together with $v$ a $G_{L T} . \diamond$

Hence, assume that no two nodes in $y y^{\prime} \in Y_{S}$ with distinct neighbors on $\bar{C}$ are adjacent (otherwise, we are done).

Claim 16 If $Z \neq \emptyset$, then $G$ contains a node stretching of $G_{E M N}$ as induced subgraph.

If there is a node $z \in Z$, then $z$ is adjacent to every node in $Y_{S}$ (otherwise, there is a node $y \in Y_{S}$ such that $z$ together with $S_{y}$ forms a stable set of size 4 ). Recall that we assume that $Y_{S}$ contains two non-adjacent nodes $y, y^{\prime}$ with distinct neighbors on $\bar{C}$. Then $z$ together with $y, y^{\prime}$ and $\bar{C}$ induce a node stretching of $G_{E M N} \vee \diamond$

Hence, we are done if $Z \neq \emptyset$ (because $G$ contains an $L S_{+}$-imperfect subgraph). So let us assume $Z=\emptyset$ from now on.

Furthermore, assume w.l.o.g. that $y$ with $N_{\bar{C}}(y)=\left\{u_{1}, u_{2}\right\}$ and $y^{\prime}$ with $N_{\bar{C}}\left(y^{\prime}\right)=\left\{u_{3}, u_{4}\right\}$ is a pair of nodes in $Y_{S}$ having distinct neighbors on $\bar{C}$. Since $G$ is facet-defining (and, thus, without clique cutset), there is a path connecting $y$ and $y^{\prime}$; let $P$ denote the shortest such path.

Note that $P$ has length $\geq 2$ (recall: $y$ and $y^{\prime}$ are supposed to be non-adjacent, otherwise $G$ contains a $G_{L T}$ by Claim 15 ).

Claim 17 If $P$ has length 2 , then $G$ is $L S_{+}$-imperfect.

So, let $P$ have length 2 and denote by $t$ its only internal node. Then $t \in Y$ follows (by $Z=\emptyset$ and because there is no common neighbor of $y$ and $y^{\prime}$ in $\bar{C}$ ). We conclude that $t \notin Y_{*}$ holds (otherwise, $G$ has a claw induced by $t$ and $S_{y}$ ) and that $t$ and $u_{5}$ are non-adjacent (otherwise, $G$ has a claw induced by $t$ and $y$, $\left.y^{\prime}, u_{5}\right)$. In fact, $N_{\bar{C}}(t)=\left\{u_{2}, u_{3}\right\}$ follows by our assumption that no two nodes in $Y_{S}$ with distinct neighbors on $\bar{C}$ are adjacent (otherwise, $G$ contains a $G_{L T}$ by Claim 15).

Since the graph induced by $G_{v}$ together with $y, t, y^{\prime}$, called a 3-gear (see Figure 7), is not facet-defining, there must be another node $y^{\prime \prime}$ in $Y$ (recall: we have $Z=\emptyset$ ). We are done if there is a node $y^{\prime \prime} \in Y_{*}$ (because $G$ contains a $G_{E M N}$ induced by $u_{1}, y, t, u_{3}, v$ and $y^{\prime \prime}$ in this case). Hence, assume $y^{\prime \prime} \in Y_{S}$.

W.l.o.g., let $N_{\bar{C}}\left(y^{\prime \prime}\right)=\left\{u_{4}, u_{5}\right\}$ (note: the case $N_{\bar{C}}\left(y^{\prime \prime}\right)=\left\{u_{1}, u_{5}\right\}$ is symmetric, and in all other cases, $G$ is still a 3 -gear with some replicated nodes, thus not facet-defining). Then $y^{\prime \prime}$ and $y^{\prime}$ are adjacent by Claim 11. If $y^{\prime \prime}$ is also adjacent to $y$ or $t$, then we are done since then $G$ contains a $G_{L T}$ by Claim 15. If $y^{\prime \prime}$ is neither adjacent to $y$ nor to $t$, then $G$ contains an $L S_{+}$-imperfect line graph induced by $u_{1}, y, t, u_{3}, v$ and $y^{\prime}, y^{\prime \prime}, u_{3}$ (being a node stretching of $G_{E M N}$ ). Note that $G$ still contains one of the above $L S_{+}$-imperfect subgraphs if $G$ contains more nodes than considered so far. $\diamond$ 


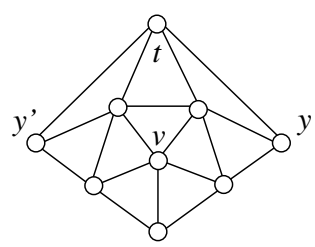

Fig. 7. Subgraph induced by $\bar{C}$ and $v, y, y^{\prime}, t$, called a 3 -gear.

Thus, we are done if $P$ has length 2 . Let us finally assume that $P$ has length $\geq$ 3 and $y$ and $y^{\prime}$ have no common neighbor (recall: $P$ is a shortest path connecting them).

Then $Y_{*}=\emptyset$ follows (because each node $y^{\prime \prime} \in Y_{*}$ shares a common neighbor with $y$ and $y^{\prime}$ on $\bar{C}$ and is, thus, adjacent to both $y$ and $y^{\prime}$ by Claim 11, a contradiction to the choice of $P$ as shortest path connecting them).

Moreover, there is a neighbor $\bar{y}$ of $y$ in $Y_{S}$ (otherwise, $\left\{u_{1}, u_{2}\right\}$ forms a clique cutset separating $y$ from $Q$, a contradiction to $G$ facet-defining by Chvátal [5]). In addition, $N_{\bar{C}}(\bar{y})$ is different from $\left\{u_{3}, u_{4}\right\}$ and from $\left\{u_{4}, u_{5}\right\}$ (otherwise, $y$ and $y^{\prime}$ would be a pair of nodes with distinct neighbors on $\bar{C}$ and connected by a path of length 2, hence $G$ is $L S_{+}$-imperfect by Claim 17 and we are done).

We clearly have $N_{\bar{C}}(\bar{y}) \neq\left\{u_{2}, u_{3}\right\}$ (otherwise, $\bar{y}$ adjacent to $y^{\prime}$ follows by Claim 11 and we have again a pair of nodes with distinct neighbors on $\bar{C}$ and connected by a path of length 2 , hence $G$ is $L S_{+}$-imperfect by Claim 17 and we are done).

If there is no node in $Y_{S}$ having $\left\{u_{1}, u_{5}\right\}$ as neighbors on $\bar{C}$, then $\left\{u_{1}, u_{2}\right\}$ forms still a clique cutset separating $y$ from $Q$, again a contradiction. Hence, let $N_{\bar{C}}(\bar{y})=\left\{u_{1}, u_{5}\right\}$.

Then, there is no node in $Y_{S}$ having $\left\{u_{4}, u_{5}\right\}$ as neighbors on $\bar{C}$ (this node $y^{\prime \prime}$ would be adjacent to $\bar{y}$ by Claim 11 , so that $\bar{y}$ would be a common neighbor of $y$ and $y^{\prime \prime}$, leading to an $L S_{+}$-imperfect subgraph of $G$ by Claim 17 and we are done). Similarly, there is no node in $Y_{S}$ having $\left\{u_{3}, u_{4}\right\}$ as neighbors on $\bar{C}$.

This finally implies that $\left\{u_{3}, u_{4}\right\}$ is a clique cutset separating $y^{\prime}$ from $Q$, a contradiction to $G$ facet-defining by Chvátal [5]). That all further nodes of $G$ are either replicates of $y, y^{\prime}$ or $\bar{y}$ (and $\left\{u_{3}, u_{4}\right\}$ remains a clique cutset in all cases) finishes the proof.

Hence, the only facet-defining subgraphs $G^{\prime}$ of $\mathrm{LS}_{+}$-perfect claw-free not quasi-line graphs $G$ with $\alpha(G)=3$ have $\alpha\left(G^{\prime}\right)=2$ and are, by Theorem 6 , cliques, odd antiholes or their complete joins. We conclude that $\mathrm{LS}_{+}$-perfect facet-defining claw-free not quasi-line graphs $G$ with $\alpha(G)=3$ are joined $a$ perfect and, thus, the $\mathrm{LS}_{+}$-Perfect Graph Conjecture is true for this class.

This together with Theorem 9 shows that the only facet-defining subgraphs $G^{\prime}$ of $\mathrm{LS}_{+}$-perfect claw-free not quasi-line graphs $G$ with $\alpha(G) \geq 4$ have $\alpha\left(G^{\prime}\right)=$ 2 and are, by Theorem [6. cliques, odd antiholes or their complete joins. Thus, 
every $\mathrm{LS}_{+}$-perfect claw-free not quasi-line graph $G$ with $\alpha(G) \geq 4$ is joined a-perfect and, thus, the $\mathrm{LS}_{+}$-Perfect Graph Conjecture holds true for this class.

Combining Corollary 1 with the above results shows that all $\mathrm{LS}_{+}$-perfect claw-free but not quasi-line graphs are joined $a$-perfect and we obtain:

Corollary 3. The $\mathrm{LS}_{+}$-Perfect Graph Conjecture is true for all claw-free graphs that are not quasi-line.

Finally, we obtain our main result (Theorem 1) as direct consequence of Corollary 2 and Corollary 3 ; The $\mathrm{LS}_{+}$-Perfect Graph Conjecture is true for all claw-free graphs.

\section{Conclusion and future research}

The context of this work was the study of $\mathrm{LS}_{+}$-perfect graphs, i.e., graphs where a single application of the Lovász-Schrijver PSD-operator $\mathrm{LS}_{+}$to the edge relaxation yields the stable set polytope. Hereby, we are particularly interested in finding an appropriate polyhedral relaxation $P(G)$ of $\operatorname{STAB}(G)$ that coincides with $\operatorname{LS}_{+}(G)$ and $\operatorname{STAB}(G)$ if and only if $G$ is $\mathrm{LS}_{+}$-perfect. An according conjecture has been recently formulated ( $\mathrm{LS}_{+}$-Perfect Graph Conjecture); here we verified it for the well-studied class of claw-free graphs (Theorem 1).

For that, it surprisingly turned out that it was not necessary to make use of the description of $\operatorname{STAB}(G)$ for claw-free not quasi-line graphs $G$

- with $\alpha(G)=2$ (by Cook, see [31]),

- with $\alpha(G)=3$ (by Pêcher, Wagler [28),

- with $\alpha(G) \geq 4$ (by Galluccio, Gentile, Ventura [131415]).

From the presented results and proofs, we can draw some further conclusions. First of all, we can determine the subclass of joined $a$-perfect graphs to which all $\mathrm{LS}_{+}$-perfect claw-free graphs belong to. In 20, it is suggested to call a graph $G$ m-perfect if the only facets of $\operatorname{STAB}(G)$ are associated with cliques and minimally imperfect graphs. According to $[\underline{6}, G$ is joined m-perfect if $\operatorname{STAB}(G)$ is given only by facets associated with cliques, minimally imperfect graphs and their complete joins. Theorem 5 together with the results from Section 3 provide the complete list of all facet-defining $\mathrm{LS}_{+}$-perfect claw-free graphs:

- cliques,

- odd holes and odd antiholes,

- complete joins of odd antihole(s) and a (possibly empty) clique.

Hence, we conclude:

Corollary 4. All $\mathrm{LS}_{+}$-perfect claw-free graphs are joined m-perfect.

Among these possible facets, only complete joins of odd antihole(s) and a non-empty clique are non-rank. This directly implies: 
Corollary 5. A rank-perfect $\mathrm{LS}_{+}$-perfect claw-free graph has as only facet-defining subgraphs cliques, odd holes, odd antiholes, or complete joins of the latter.

Note that Galluccio and Sassano provided in [16] a complete characterization of the rank facet-defining claw-free graphs: they either belong to one of the following three families of rank-minimal graphs

- cliques,

- partitionable webs $W_{\alpha \omega+1}^{\omega-1}$ (where $\alpha$ and $\omega$ stand for stability and clique number, resp.),

- line graphs of minimal 2-connected hypomatchable graphs $H$ (where $H-e$ is not hypomatchable anymore for any edge $e$ ),

or can be obtained from them by means of two operations, sequential lifting and complete join. Our results show: an $\mathrm{LS}_{+}$-perfect claw-free graph $G$ has, besides cliques, only odd holes and odd antiholes as rank-minimal subgraphs; cliques are the only subgraphs in $G$ that can be sequentially lifted to larger rank facetdefining subgraphs, where complete joins can only be taken of odd antiholes.

Note further that, besides verifying the $\mathrm{LS}_{+}$-Perfect Graph Conjecture for claw-free graphs, we obtained the complete list of all minimally $\mathrm{LS}_{+}$-imperfect claw-free graphs. In fact, the results in [111/2 imply that the following graphs are minimally $\mathrm{LS}_{+}$-imperfect:

- graphs $G$ with $\alpha(G)=2$ such that $G-v$ is an odd antihole for some node $v$, not completely joined to $G-v$,

- the web $W_{10}^{2}$,

- $\mathrm{LS}_{+}$-imperfect line graphs (which are all node stretchings of $G_{L T}$ or $G_{E M N}$ ).

Our results from Section 3 on facet-defining $\mathrm{LS}_{+}$-perfect claw-free graphs imply that they are the only minimally $\mathrm{LS}_{+}$-imperfect claw-free graphs.

Finally, the subject of the present work has parallels to the well-developed research area of perfect graph theory also in terms of polynomial time computability. In fact, it has the potential of reaching even stronger results due the following reasons. Recall that calculating the value

$$
\eta_{+}(G)=\max \mathbf{1}^{T} x, x \in \mathrm{LS}_{+}(G)
$$

can be obtained with arbitrary precision in polynomial time for every graph $G$, even in the weighted case, by [24. Thus, the stable set problem can be solved in polynomial time for a strict superset of perfect graphs, the $\mathrm{LS}_{+}$-perfect graphs, by $\alpha(G)=\eta_{+}(G)$. Hence, our future lines of research include to find

- new families of graphs where the conjecture holds (e.g., by characterizing the minimally $\mathrm{LS}_{+}$-imperfect graphs within the class),

- new subclasses of $\mathrm{LS}_{+}$-perfect or joined a-perfect graphs,

- classes of graphs $G$ where $\operatorname{STAB}(G)$ and $\mathrm{LS}_{+}(G)$ are "close enough" to have $\alpha(G)=\left\lfloor\eta_{+}(G)\right\rfloor$. 
In particular, the class of graphs $G$ with $\alpha(G)=\left\lfloor\eta_{+}(G)\right\rfloor$ can be expected to be large since $\mathrm{LS}_{+}(G)$ is a much stronger relaxation of $\operatorname{STAB}(G)$ than $\operatorname{TH}(G)$. In all cases, the stable set problem could be approximated with arbitrary precision in polynomial time in these graph classes by optimizing over $\mathrm{LS}_{+}(G)$. Finally, note that $\mathrm{LS}_{+}(P(G))$ with

$$
\operatorname{STAB}(G) \subseteq P(G) \subseteq \operatorname{ESTAB}(G)
$$

clearly gives an even stronger relaxation of $\operatorname{STAB}(G)$ than $\mathrm{LS}_{+}(G)$. However, already approximating with arbitrary precision over $\operatorname{LS}_{+}(\operatorname{QSTAB}(G))$ cannot be done in polynomial time anymore for all graphs $G$ by 24]. Hence, $\mathrm{LS}_{+-}$ perfect graphs or their generalizations satisfying $\alpha(G)=\left\lfloor\eta_{+}(G)\right\rfloor$ are the most promising cases in this context.

\section{References}

1. S. Bianchi, M. Escalante, G. Nasini, and L. Tunçel, "Near-perfect graphs with polyhedral $N_{+}(G)$," Electronic Notes in Discrete Math., vol. 37, pp. 393-398, 2011.

2. S. Bianchi, M. Escalante, G. Nasini, and L. Tunçel, "Lovász-Schrijver PSDoperator and a superclass of near-perfect graphs", Electronic Notes in Discrete Mathematics, vol. 44, pp. 339-344, 2013.

3. M. Chudnovsky, N. Robertson, P. Seymour, and R. Thomas, "The Strong Perfect Graph Theorem," Annals of Mathematics, vol. 164, pp. 51-229, 2006.

4. M. Chudnovsky and P. Seymour, The structure of claw-free graphs, manuscript (2004).

5. V. Chvátal, "On Certain Polytopes Associated with Graphs," J. Combin. Theory (B), vol. 18, pp. 138-154, 1975.

6. S. Coulonges, A. Pêcher, and A. Wagler, "Characterizing and bounding the imperfection ratio for some classes of graphs," Math. Programming A, vol. 118, pp. 37-46, 2009.

7. J.R. Edmonds, "Maximum Matching and a Polyhedron with $(0,1)$ Vertices," J. Res. Nat. Bur. Standards, vol. 69B, pp. 125-130, 1965.

8. J.R. Edmonds and W.R. Pulleyblank, "Facets of 1-Matching Polyhedra," In: C. Berge and D.R. Chuadhuri (eds.) Hypergraph Seminar. Springer, pp. 214-242, 1974.

9. F. Eisenbrand, G. Oriolo, G. Stauffer, and P. Ventura, The stable set polytope of quasi-line graphs. Combinatorica, 28:45-67, 2008.

10. M. Escalante, M.S. Montelar, and G. Nasini, "Minimal $N_{+}$-rank graphs: Progress on Lipták and Tunçel's conjecture," Operations Research Letters, vol. 34, pp. 639 646, 2006.

11. M. Escalante and G. Nasini, "Lovász and Schrijver $N_{+}$-relaxation on web graphs," Lecture Notes in Computer Sciences, vol. 8596 (ISCO 2014), pp. 221-229, 2014.

12. M. Escalante, G. Nasini, and A. Wagler, "Characterizing $N_{+}$-perfect line graphs", International Transactions in Operational Research, 2015, DOI: 10.1111 /itor. 12275 .

13. A. Galluccio, C. Gentile, and P. Ventura. Gear composition and the stable set polytope. Operations Research Letters, 36:419-423, 2008.

14. A. Galluccio, C. Gentile, and P. Ventura. The stable set polytope of claw-free graphs with stability number at least four. I. Fuzzy antihat graphs are W-perfect, Journal of Combinatorial Theory Series B 107, 92-122, 2014 
15. A. Galluccio, C. Gentile, and P. Ventura. The stable set polytope of claw-free graphs with stability number at least four. II. Striped graphs are G-perfect, Journal of Combinatorial Theory Series B 108, 1-28, 2014

16. A. Galluccio and A. Sassano, The Rank Facets of the Stable Set Polytope for ClawFree Graphs. J. Comb. Theory B 69 (1997) 1-38.

17. R. Giles and L.E. Trotter. On stable set polyhedra for K1,3 -free graphs. J. of Combinatorial Theory, 31:313-326, 1981.

18. M. Grötschel, L. Lovász, and A. Schrijver, "The Ellipsoid Method and its Consequences in Combinatorial Optimization," Combinatorica, vol. 1, pp. 169-197, 1981.

19. M. Grötschel, L. Lovász, and A. Schrijver, Geometric Algorithms and Combinatorial Optimization. Springer-Verlag (1988)

20. A. Koster and A. Wagler, Comparing imperfection ratio and imperfection index for graph classes, RAIRO Operations Research, vol. 42, pp. 485-500, 2008.

21. T.M. Liebling, G. Oriolo, B. Spille, and G. Stauffer. On non-rank facets of the stable set polytope of claw-free graphs and circulant graphs. Mathematical Methods of Operations Research, 59(1):25-35, 2004.

22. L. Lipták and L. Tunçel, "Stable set problem and the lift-and-project ranks of graphs," Math. Programming A, vol. 98, pp. 319-353, 2003.

23. L. Lovász, "On the Shannon capacity of a graph," IEEE Trans. Inf. Theory, vol. 25, pp. 1-7, 1979.

24. L. Lovász and A. Schrijver, "Cones of matrices and set-functions and 0-1 optimization," SIAM J. on Optimization, vol. 1, pp. 166-190, 1991.

25. G. Minty, "On maximal independent sets of vertices in claw-free graphs", J. of Combinatorial Theory, vol. 28, pp. 284-304, 1980.

26. G. Oriolo, U. Pietropaoli, and G. Stauffer, "A new algorithm for the maximum weighted stable set problem in claw-free graphs", in A. Lodi, A. Panconesi and G. Rinaldi (eds), IPCO 2008, Vol. 5035 of Lecture Notes in Computer Science, Springer, Bertinoro, Italy, May 26-28, pp. 77-96, 2008.

27. A. Pêcher, A. Wagler, "Almost all webs are not rank-perfect", Math. Programming B 105 (2006) 311-328

28. A. Pêcher, A. Wagler, "On facets of stable set polytopes of claw-free graphs with stability number three", Discrete Mathematics 310 (2010) 493-498

29. U. Pietropaoli, A. Wagler, "Some results towards the description of the stable set polytope of claw-free graphs", Proc. of ALIO/EURO Workshop on Applied Combinatorial Optimization, Buenos Aires, 2008

30. N. Sbihi, "Algorithme de recherche d'un stable de cardinalité maximum dans un graphe sans étoile", Discrete Mathematics 29, 53-76, 1980.

31. F.B. Shepherd, "Near-Perfect Matrices," Math. Programming, vol. 64, pp. 295-323, 1994.

32. F.B. Shepherd, "Applying Lehman's Theorem to Packing Problems," Math. Programming, vol. 71, pp. 353-367, 1995.

33. G. Stauffer, "On the Stable set polytope of claw-free graphs". Phd thesis, 2005, Swiss Institute of Technology in Lausanne.

34. A. Wagler, Critical Edges in Perfect Graphs. PhD thesis, TU Berlin and Cuvillier Verlag Göttingen (2000).

35. A. Wagler, "Antiwebs are rank-perfect," 4OR, vol. 2, pp. 149-152, 2004.

36. A. Wagler, "On rank-perfect subclasses of near-bipartite graphs," 4OR, vol. 3, pp. 329-336, 2005. 